Received: 13 February 2017

Accepted: 17 May 2017

Published online: 26 June 2017

\section{OPEN The Incidence, Risk Factors and Outcomes of Postoperative Acute Kidney Injury in Neurosurgical Critically III Patients}

Yujun Deng ${ }^{1}$, Jie Yuan ${ }^{1}$, Ruibin $\mathrm{Chi}^{2}$, Heng Ye ${ }^{3}$, Dong Zhou ${ }^{4}$, Sheng Wang ${ }^{5}$, Cong Mai ${ }^{1}$, Zhiqiang Nie ${ }^{6}$, Lin Wang ${ }^{1}$, Yiling Zhai ${ }^{1}$, Lu Gao ${ }^{1}$, Danqing Zhang ${ }^{1}$, Linhui Hu ${ }^{1}$, Yiyu Deng ${ }^{1}$ \& Chunbo Chen ${ }^{1}$

We investigated the incidence, perioperative risk factors, and outcomes of postoperative acute kidney injury (AKI) in neurosurgical critically ill patients. A prospective multicenter cohort study was conducted, enrolling adult patients who underwent neurosurgical procedure and admitted to the neurosurgical intensive care units (ICU). Postoperative AKI was diagnosed within 7 days after surgery based on the Kidney Disease Improving Global Outcomes criteria. Of 624 enrolled patients, postoperative AKI occurred in 84 patients. AKI was associated with increased rates of ICU and in-hospital mortality, postoperative renal replacement therapy, postoperative tracheotomy, and postoperative tracheal reintubation. Patients who developed AKI had higher total ICU costs, prolonged length of hospital and ICU stay, and longer duration of postoperative mechanical ventilation. Multivariate analysis identified postoperative reoperation (adjusted odds ratio [OR] $5.70[95 \% \mathrm{Cl}$, 1.61-20.14]), postoperative concentration of serum cystatin C (adjusted OR 4.53 [95\% Cl, 1.98-10.39]), use of mannitol during operation (adjusted OR 1.97 [95\% Cl, 1.13-3.43]), postoperative APACHE II score (adjusted OR 1.11 [95\% Cl, 1.06-1.16]), and intraoperative estimated blood loss (adjusted OR 1.04 [95\% $\mathrm{Cl}, 1.00-1.08]$ ) as independent risk factors for postoperative AKI. Postoperative AKI in neurosurgical critically ill cohort is prevalent and associated with adverse in-hospital outcomes.

Postoperative acute kidney injury (AKI) is a highly prevalent and prognostically important complication in various surgical settings. Patients who developed postoperative AKI is independently associated with markedly increased morbidity, mortality ${ }^{1-8}$ and higher economic burden ${ }^{1}$. A considerable amount of publications have evaluated the incidence, determinants, and consequences of AKI in patients undergoing cardiac surgery $\mathrm{y}^{4,9,10}$ or non-cardiac surgery $2,5,11-13$. Consequently, several preventive and treatment strategies have been developed. Nevertheless, little is known about the incidence, risk factors and outcomes of postoperative AKI in neurosurgical critically ill patients, and hence may lead to an unacceptable delay in initiating any therapy regimens.

A better knowledge of the clinical characteristics of postoperative AKI in neurosurgical critically ill cohort may help develop efficacious intervention of this complication. The aims of this study were to demonstrate the incidence of postoperative AKI in neurosurgical critically ill population, identify perioperative risk factors, and clarify the relationship between AKI and in-hospital outcomes.

${ }^{1}$ Department of Critical Care Medicine, Guangdong General Hospital, Guangdong Academy of Medical Sciences, Guangzhou, 510080, Guangdong Province, P.R. China. ${ }^{2}$ Department of Critical Care Medicine, Xiaolan Hospital of Southern Medical University, Zhongshan, 528415, Guangdong, P.R. China. ${ }^{3}$ Department of Critical Care Medicine, Guangzhou Nansha Central Hospital, Nansha, 511400, Guangdong, P.R. China. 'Department of Neurosurgery, Guangdong General Hospital, Guangdong Academy of Medical Sciences, Guangzhou, Guangdong, 510080, P.R. China. ${ }^{5}$ Department of Anesthesiology, Guangdong Cardiovascular Institute and Guangdong General Hospital, Guangdong Academy of Medical Sciences, Guangzhou, Guangdong, 510080, P.R. China. ${ }^{6}$ Department of Cardiovascular Epidemiology, Cardiac Surgery, Guangdong Cardiovascular Institute, Guangdong General Hospital, Guangdong Academy of Medical Sciences, Guangzhou, Guangdong, 510080, P.R. China. Yujun Deng and Jie Yuan contributed equally to this work. Correspondence and requests for materials should be addressed to Y.D. (email: yiyudeng666@163.com) or C.C. (email: gghccm@163.com) 


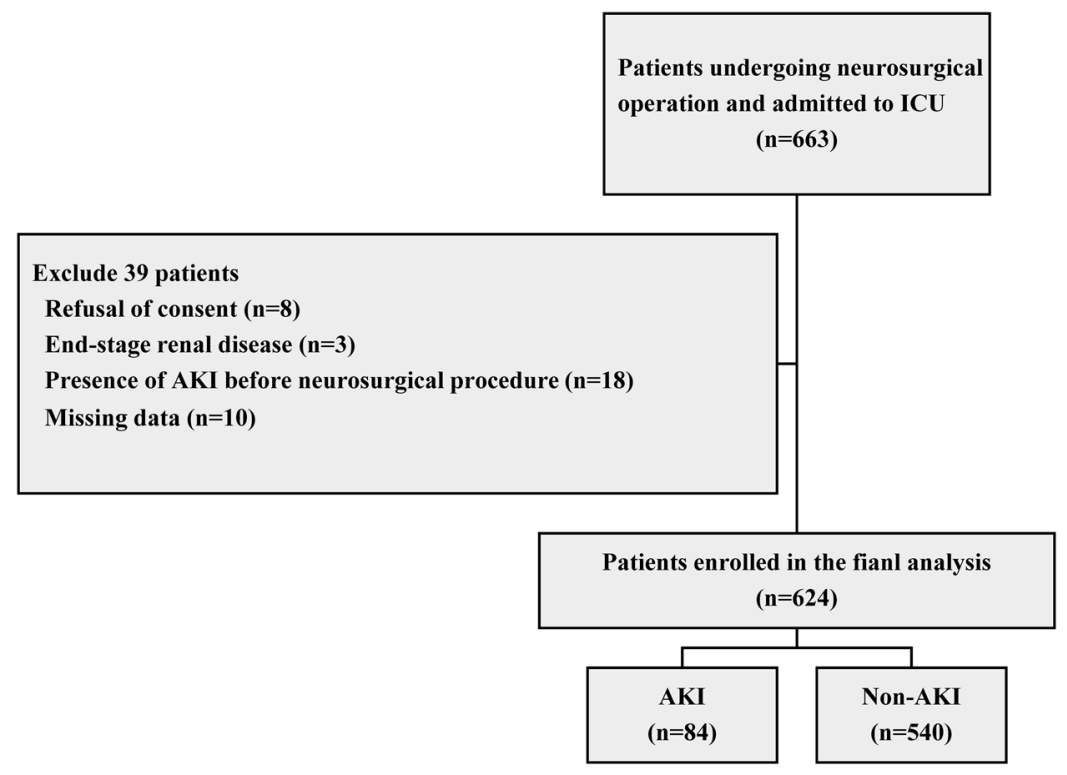

Figure 1. Flow chart from recruitment to outcome. Abbreviations: ICU, intensive care unit; AKI, acute kidney injury.

\section{Results}

Patient's preoperative characteristics. Of the 663 consecutive patients who were screened for inclusion in the study, 39 (5.9\%) were excluded (Fig. 1). 624 patients were enrolled for analysis. Table 1 presents preoperative characteristics of patients. Overall, AKI based on the Kidney Disease Improving Global Outcomes (KDIGO) criteria occurred in 84 patients (13.5\%) within the first 7 days after neurosurgical operation. Of these AKI patients, 72 patients $(85.7 \%)$ were at stage 1,8 patient $(9.5 \%)$ at stage 2 , and 4 patients at stage $3(4.8 \%)$. Among the 84 AKI patients, 14 developed AKI in the first day after operation, 57 in the second day, 10 in the third day, and 3 patients beyond 3 days. Thus, 96.4\% of the patients reached AKI within 3 days after operation. Preexisting chronic kidney disease (CKD) was more frequent in patients with AKI. AKI patients had higher rate of emergency surgery when compared with non-AKI patients. Moreover, patients with AKI had higher American Society of Anesthesiologists (ASA) classification. However, there are no significant differences in age, sex, body mass index (BMI), baseline serum creatinine, baseline estimated glomerular filtration rate (eGFR), and preoperative hemoglobin concentration between patients with and without AKI. Additionally, AKI patients took no more nephrotoxic drugs preoperatively than non-AKI patients. There was also no significant difference of preoperative application of radiographic contrast or mannitol between AKI and non-AKI patients. Most of the patients (73.9\%) underwent operation for intracranial tumor.

Patient's intraoperative characteristics. Table 2 demonstrates intraoperative parameters in this cohort. Intraoperative estimated blood loss in patients undergoing postoperative AKI was more than that in non-AKI patients. In addition, the percentage of patients who received mannitol or blood transfusion (platelets) during operation was significantly higher in AKI patients than in non-AKI patients. However, patients with AKI were not treated with more crystalloid and colloid (including hydroxyethyl starch) during operation in comparison with patients without AKI.

Patient's postoperative characteristics. Patients who experienced AKI were likely to have lower hemoglobin levels after operation, higher concentrations of postoperative serum cystatin $\mathrm{C}$ (CysC) and serum creatinine $(\mathrm{sCr})$ than patients without AKI (Table 3). Furthermore, they were more likely to undergo reoperation after the first neurosurgical procedure. The severity of disease scores [the postoperative Acute Physiology and Chronic Health Evaluation score (APACHE II) and Glasgow Coma Scale (GCS) score] for patients were also showed. Compared with patients without AKI, patients with AKI were more severely ill.

Multivariable analysis of risk factors that are related to postoperative AKI. Risk factors which are significantly correlated with the incidence of postoperative AKI are depicted in Table 4. The independent risk factors for postoperative AKI included estimated blood loss during operation, postoperative reoperation, use of mannitol during operation, concentration of postoperative serum CysC, and postoperative APACHE II score. Intraoperative estimated blood loss may increase the rate of AKI occurrence in an amount-dependent manner, with each $100 \mathrm{ml}$ estimated blood loss increasing the odds of AKI 1.04-fold. The risk for developing AKI was 5.70 times higher in reoperation patients. Each unit increment in postoperative serum $\mathrm{Cys} C$ was independently associated with AKI (adjusted odds ratio [OR] 4.53 [95\% CI, 1.98-10.39]) after adjustment for clinical covariates. Additional, the risk for postoperative AKI was 1.97 times higher in those used mannitol during operation. We also found that higher postoperative APACHE II score could predict AKI occurrence (adjusted OR 1.11 [95\% CI, $1.06-1.16])$. 


\begin{tabular}{|c|c|c|c|c|}
\hline Characteristics & All patients $(n=624)$ & Non-AKI $(n=540)$ & AKI $(n=84)$ & $P$ value \\
\hline Age, years & $50(40,60)$ & $50(40,59)$ & $54(39,65)$ & 0.056 \\
\hline Gender (male), n (\%) & $283(45.4)$ & $244(45.2)$ & $40(46.4)$ & 0.831 \\
\hline $\mathrm{BMI}, \mathrm{kg} / \mathrm{m}^{2}$ & $22.5(20.0,25.2)$ & $22.3(19.9,25.2)$ & $22.8(20.1,24.6)$ & 0.845 \\
\hline \multicolumn{5}{|l|}{ Preexisting clinical conditions, n (\%) } \\
\hline Hypertension & $91(14.6)$ & $79(14.6)$ & $12(14.3)$ & 0.934 \\
\hline Diabetes mellitus & $34(5.4)$ & $28(5.2)$ & $6(7.1)$ & 0.440 \\
\hline CKD & $12(1.9)$ & $6(1.1)$ & $6(7.1)$ & 0.002 \\
\hline Cerebrovascular disease & $32(5.1)$ & $27(5.0)$ & $5(6.0)$ & 0.789 \\
\hline Hyperlipidemia & $20(3.2)$ & $15(2.8)$ & $5(6.0)$ & 0.171 \\
\hline CAD & $9(1.4)$ & $6(1.1)$ & $3(3.6)$ & 0.108 \\
\hline Prior neurological surgery, $\mathrm{n}(\%)$ & $54(8.7)$ & $45(8.3)$ & $9(10.7)$ & 0.470 \\
\hline Emergency surgery, $\mathrm{n}(\%)$ & $54(8.7)$ & $40(7.4)$ & $14(16.7)$ & 0.005 \\
\hline ASA classification, $\mathrm{n}(\%)$ & & & & 0.018 \\
\hline I & $431(69.1)$ & $382(70.7)$ & $49(58.3)$ & \\
\hline II & $118(18.9)$ & $101(18.7)$ & $17(20.2)$ & \\
\hline III & $27(4.3)$ & $23(4.3)$ & $4(4.8)$ & \\
\hline IV & $42(6.7)$ & $30(5.6)$ & $12(14.3)$ & \\
\hline $\mathrm{V}$ & $6(1.0)$ & $4(0.7)$ & $2(2.4)$ & \\
\hline Preoperative hemoglobin, g/L & $130(119,141)$ & $130(119,141)$ & $129(119,141)$ & 0.761 \\
\hline Baseline serum creatinine, $\mathrm{mg} / \mathrm{dl}$ & $0.69(0.59,0.84)$ & $0.69(0.59,0.84)$ & $0.68(0.53,0.91)$ & 0.704 \\
\hline Baseline eGFR, $\mathrm{ml} / \mathrm{min} / 1.73 \mathrm{~m}^{2}$ & $106.5(94.8,117.3)$ & $106.5(95.1,117.3)$ & $106.7(87.4,120.3)$ & 0.488 \\
\hline \multicolumn{5}{|l|}{ Preoperative medication, $\mathrm{n}(\%)$} \\
\hline Nephrotoxic drugs ${ }^{\mathrm{b}}$ & $18(2.9)$ & $15(2.8)$ & $3(3.6)$ & 0.723 \\
\hline Radiographic contrast & $26(4.2)$ & $22(4.1)$ & $4(4.8)$ & 0.768 \\
\hline Mannitol & $102(16.3)$ & $86(15.9)$ & $16(19.0)$ & 0.472 \\
\hline Diagnostic group, n (\%) & & & & 0.169 \\
\hline Spine disease & $32(5.1)$ & $31(5.7)$ & $1(1.2)$ & \\
\hline Hydrocephalus & $22(3.5)$ & $19(3.5)$ & $3(3.6)$ & \\
\hline Intracranial tumor & $461(73.9)$ & $399(73.9)$ & $62(73.8)$ & \\
\hline Traumatic brain injury & $16(2.6)$ & $12(2.2)$ & $4(4.8)$ & \\
\hline Intracranial aneurysm/AVM & $27(4.3)$ & $22(4.1)$ & $5(6.0)$ & \\
\hline Hypertensive cerebral hemorrhage & $10(1.6)$ & $7(1.3)$ & $3(3.6)$ & \\
\hline Others & $56(9.0)$ & $50(9.3)$ & $6(7.1)$ & \\
\hline
\end{tabular}

Table 1. Preoperative Patient Characteristics by Status of $A K I^{a}$. ${ }^{a}$ Continuous variables were expressed as mean \pm SD or median (25th percentile-75th percentile, IQR). Categorical variables were expressed as a number (\%). 'Includes any of the following medications administered within 5 days before operation: nonsteroidal antiinflammatory drug, angiotensin-converting enzyme inhibitor, angiotensin receptor blocker, immunosuppressant, aminoglycoside, vancomycin, acyclovir, amphotericin. Abbreviations: AKI, acute kidney injury; BMI, body mass index; CKD, chronic kidney disease; CAD, coronary artery disease; ASA classification, American Society of Anesthesiologists classification; eGFR, estimated glomerular filtration rate; AVM, arteriovenous malformation.

In-hospital outcomes and postoperative AKI. In the present study, AKI occurrence was associated with higher rates of postoperative renal replacement therapy (RRT), intensive care unit (ICU) mortality, in-hospital mortality, postoperative tracheotomy, and tracheal reintubation (Table 5). Moreover, patients with AKI had higher total ICU costs, prolonged hospital and ICU length of stay, and longer duration of postoperative mechanical ventilation.

\section{Discussion}

In the multi-center prospective study, AKI occurred frequently and was associated with adverse in-hospital outcomes in neurosurgical critically ill population. The independent risk factors of postoperative AKI occurrence included intraoperative estimated blood loss, postoperative reoperation, use of mannitol during operation, concentration of postoperative serum CysC, and postoperative APACHE II score.

Concomitant with social and economic development, the number of neurosurgical operations increased worldwide ${ }^{14,15}$ and great progress has been made in neurosurgery. Although a retrospective study reported the incidence of AKI after craniotomy in a large cohort was $9.9 \%{ }^{16}$. Prospective study about the incidence, determinants and consequences of postoperative AKI in entire neurosurgical critically ill patients is scarce. Therefore, we conducted a prospective study to assess the incidence, risk factors and outcomes of AKI occurrence in such a relatively large multicenter neurosurgical cohort. 


\begin{tabular}{|l|l|l|l|l|}
\hline & $\begin{array}{l}\text { All patients } \\
(\mathbf{n = 6 2 4})\end{array}$ & $\begin{array}{l}\text { Non-AKI } \\
(\mathbf{n}=\mathbf{5 4 0})\end{array}$ & AKI (n=84) & P value \\
\hline General anesthesia, n (\%) & $620(99.4)$ & $537(99.4)$ & $83(98.8)$ & 0.444 \\
\hline Duration of surgery, minute & $290(190,380)$ & $285(185,370)$ & $300(210,410)$ & 0.172 \\
\hline Estimated blood loss, per 100 ml & $2.0(1.0,4.4)$ & $2.0(1.0,4.0)$ & $3.0(1.5,6.0)$ & 0.022 \\
\hline Minimum MAP, mm Hg & $73.3(67.3,78.7)$ & $73.3(67.7,78.6)$ & $72.5(66.8,79.8)$ & 0.719 \\
\hline Radiographic contrast, n (\%) & $12(1.9)$ & $11(2.0)$ & $1(1.2)$ & 1.000 \\
\hline Intraoperative fluids & \multicolumn{5}{|l}{} \\
\hline Total crystalloid, per 500 ml & $3.0(2.0,4.4)$ & $3.0(2.0,4.4)$ & $3.1(2.0,4.2)$ & 0.925 \\
\hline Total colloid, per 500 ml & $2.0(1.0,2.0)$ & $2.0(1.0,2.0)$ & $2.0(1.0,2.5)$ & 0.077 \\
\hline Volume of HES, per 500 ml & $1.0(0.5,2.0)$ & $1.0(0.5,2.0)$ & $1.0(0.0,2.0)$ & 0.889 \\
\hline Mannitol, n (\%) & $124(19.9)$ & $97(18.0)$ & $27(32.1)$ & 0.002 \\
\hline RBCs, n (\%) & $119(19.1)$ & $97(18.0)$ & $22(26.2)$ & 0.074 \\
\hline Plasma, n (\%) & $62(9.9)$ & $53(9.8)$ & $9(10.7)$ & 0.798 \\
\hline Platelets, $\mathrm{n}(\%)$ & $6(1.0)$ & $3(0.6)$ & $3(3.6)$ & 0.035 \\
\hline
\end{tabular}

Table 2. Intraoperative Variables by Status of $A K I^{a} .{ }^{a}$ Continuous variables were expressed as mean $\pm S D$ or median (25th percentile-75th percentile, IQR). Categorical variables were expressed as a number (\%). Abbreviations: AKI, acute kidney injury; MAP, mean arterial pressure; HES, hydroxyethyl starch; RBC, red blood cell.

\begin{tabular}{|l|l|l|l|l|}
\hline Variables & $\begin{array}{l}\text { All patients } \\
(\mathbf{n}=\mathbf{6 2 4})\end{array}$ & $\begin{array}{l}\text { Non-AKI } \\
(\mathbf{n}=\mathbf{5 4 0})\end{array}$ & AKI $(\mathbf{n = 8 4 )}$ & $\boldsymbol{P}$ value \\
\hline APACHE II score & $8(7,11)$ & $8(7,11)$ & $11(8,15)$ & $<0.001$ \\
\hline GCS score & $15(15,15)$ & $15(15,15)$ & $15(13,15)$ & $<0.001$ \\
\hline Serum Cr, mg/dl & $0.75(0.63,0.92)$ & $0.74(0.63,0.91)$ & $0.81(0.65,1.00)$ & 0.037 \\
\hline Serum CysC, mg/L & $0.73(0.62,0.87)$ & $0.73(0.61,0.86)$ & $0.81(0.67,1.03)$ & $<0.001$ \\
\hline Hemoglobin, g/L & $114.48 \pm 0.74$ & $115.29 \pm 0.80$ & $109.27 \pm 1.86$ & 0.005 \\
\hline UP, ml/kg/h & $2.2(1.6,2.9)$ & $2.2(1.6,2.8)$ & $2.4(1.7,3.4)$ & 0.102 \\
\hline Postoperative reoperation, $\mathrm{n}(\%)$ & $12(1.9)$ & $6(1.1)$ & $6(7.1)$ & 0.002 \\
\hline
\end{tabular}

Table 3. Postoperative Variables by Status of $A K I^{\mathrm{a}}$. ${ }^{\mathrm{a}}$ Continuous variables were expressed as mean $\pm \mathrm{SD}$ or median (25th percentile-75th percentile, IQR). Categorical variables were expressed as a number (\%). Abbreviations: AKI, acute kidney injury; APACHE II, Acute Physiology and Chronic Health Evaluation; GCS, Glasgow Coma Score; Cr, creatinine, CysC, Cystatin C; UP, urine production within the first 24 hours after operation; Postoperative reoperation, underwent the second neurosurgical operation within 7 days after first neurosurgical procedure.

\begin{tabular}{|l|l|l|l|l|}
\hline Variable & ORunadj & ORadj & 95\% CI & P value \\
\hline Estimated blood loss during operation (per 100 ml) & 1.04 & 1.04 & $1.00-1.08$ & 0.052 \\
\hline Postoperative reoperation & 6.85 & 5.70 & $1.61-20.14$ & 0.007 \\
\hline Postoperative sCysC (per mg/L) & 4.56 & 4.53 & $1.98-10.39$ & $<0.001$ \\
\hline Use of mannitol during operation & 2.16 & 1.97 & $1.13-3.43$ & 0.016 \\
\hline Postoperative APACHE II score & 1.13 & 1.11 & $1.06-1.16$ & $<0.001$ \\
\hline
\end{tabular}

Table 4. Multivariable Logistic Regression Analysis of Factors that Are Related to Postoperative AKI in Neurosurgical Critically Ill Patients. Abbreviations: AKI, acute kidney injury; ORunadj, odds ratio unadjusted; ORadj, odds ratio adjusted; CI, confidence interval; Postoperative reoperation, reoperation within 7 days after the first neurosurgical operation; APACHE II, Acute Physiology and Chronic Health Evaluation; sCysC, serum Cystatin C.

Our study showed that the rate of AKI occurrence was up to $13.5 \%$ in patients undergoing neurological surgery within the first 7 days. The incidence of AKI in our study is similar to those reported among several non-cardiac surgery cohorts, which varied from $7.5 \%$ to $24 \%^{2,5,12,13}$, but lower than those in cardiac surgery ${ }^{4}$, 17. Although several studies had reported the incidence of AKI in traumatic brain injury cohorts, ranging from $8 \%$ to $25 \%{ }^{18-21}$, the postoperative AKI incidence of entire neurosurgical critically ill cohort has not been well described. Our investigation provided more evidences regarding incidence of postoperative AKI in such specific population.

The risk factors of postoperative AKI varied in different clinical settings, and 5 aforementioned risk factors were identified in this neurosurgical cohort. In previous studies ${ }^{3,22}$, it has been reported that AKI was significantly related to the estimated blood loss during operation. In the present study, intraoperative estimated blood loss 


\begin{tabular}{|l|l|l|l|l|}
\hline Outcomes & $\begin{array}{l}\text { All patients } \\
(\mathbf{n = 6 2 4 )}\end{array}$ & $\begin{array}{l}\text { Non-AKI } \\
(\mathbf{n = 5 4 0 )}\end{array}$ & AKI $(\mathbf{n = 8 4 )}$ & P value \\
\hline $\begin{array}{l}\text { Duration of mechanical } \\
\text { ventilation, hours }\end{array}$ & $3(1,6)$ & $3(1,5)$ & $5(3,20)$ & $<0.001$ \\
\hline Reintubation, n (\%) & $19(3.0)$ & $10(1.9)$ & $9(10.7)$ & $<0.001$ \\
\hline Tracheotomy, n (\%) & $19(3.0)$ & $11(2.0)$ & $8(9.5)$ & 0.002 \\
\hline RRT, n (\%) & $2(0.3)$ & $0(0)$ & $2(2.4)$ & 0.018 \\
\hline ICU mortality, n (\%) & $13(2.1)$ & $2(0.4)$ & $11(13.1)$ & $<0.001$ \\
\hline Hospital mortality, n (\%) & $15(2.4)$ & $2(0.4)$ & $13(15.5)$ & $<0.001$ \\
\hline ICU length of stay, days & $1(1,2)$ & $1(1,2)$ & $2(1,3)$ & $<0.001$ \\
\hline Hospital length of stay, days & $14(10,18)$ & $13(10,18)$ & $15(11,23)$ & 0.029 \\
\hline Total ICU costs, CNY & $8888(6959,13470)$ & $8549(6894,12758)$ & $11658(8126,23985)$ & $<0.001$ \\
\hline
\end{tabular}

Table 5. Postoperative Clinical Outcomes by Status of $A K{ }^{a} .{ }^{a}$ Continuous variables were expressed as mean \pm SD or median (25th percentile-75th percentile, IQR); Categorical variables were expressed as a number (\%). Abbreviations: AKI, acute kidney injury; RRT, renal replacement therapy ICU, intensive care unit; CNY, Chinese yuan.

was the independent risk factor of postoperative AKI occurrence. On one hand, excessive bleeding may lead to hemodynamic compromise and hemoglobin reduction. Anemia, caused by significant hemoglobin reduction, can reduce renal oxygen delivery, promote oxidative stress, and impair hemostasis, ${ }^{4}$, which would contribute to AKI occurrence. On the other hand, excessive bleeding may necessitate RBC (red blood cell) transfusions. Owing to progressive structural and functional changes of preserved RBCs during storage, transfused stored RBCs may weaken tissue oxygen delivery, promote a proinflammatory state, and exacerbate tissue oxidative stress ${ }^{24}$, which are also associated with AKI. Intraoperative hypovolemia which is related to intraoperative blood loss or inadequate fluids therapy may contribute to AKI development. However, continuous monitoring volume status has not been routinely conducted during neurosurgical procedure. Therefore, the value of volume status monitoring in high-risk group need to be confirmed with respect to postoperative AKI in neurosurgical cohort.

In our study, reoperation is another determinant for postoperative AKI. Our result is consistent with previous studies $^{4,25}$, which reported that reoperation was independently associated with AKI. Although the mechanisms of AKI caused by reoperation have not been fully clarified, the logical assumption is that they involve exacerbation of many of the factors, such as hemodynamic compromise, bleeding and operative trauma, which are related to AKI occurrence.

CysC is a low molecular weight protein $(13 \mathrm{kDa})^{26}$, and considered as a reliable, functional marker for renal function. Recent studies indicated that $\mathrm{Cys} C$ is an early predictor of $\mathrm{AKI}^{27,28}$ and also has reasonable discrimination for adverse outcomes ${ }^{29}$. A previous study found ${ }^{30}$ that the increase of serum CysC preceded that of creatinine for 1-2 days in acute kidney failure detection. In the present study, the concentration of postoperative serum CysC at ICU admission, instead of serum creatinine, was an independent risk factor of postoperative AKI. Although the postoperative level of serum creatinine was associated with increased risk of AKI on univariate analysis, this relationship did not persist with multivariable adjustment. It is worthy of note that both serum CysC and creatinine are clinically available biomarkers in China and abroad, our result implies serum CysC may be a better renal biomarker for AKI prediction than serum creatinine in such population.

As many previous studies have reported the close association between mannitol and kidney injury ${ }^{31-33}$, we evaluated the relation between this drug and AKI. Our study demonstrated that use of mannitol during operation, instead of preoperative use, was an independent risk factor for postoperative AKI with a 1.97-fold increase in the risk-adjusted odds. Although the mechanisms that the use of mannitol is close associated with AKI occurrence are not completely elucidated, its possible reason is that use of mannitol might lead to swelling of proximal tubular cells and vacuolization. Undoubtedly, this would result in deterioration of kidney function ${ }^{31}$. In addition to the toxic impact on kidney, the association of AKI with intraoperative use of mannitol may also be attributed to illness severity such as high intracranial pressure or cerebral edema. Generally, intraoperative use of mannitol signifies more serious neuropathophysiological insults which are associated with high risk of $\mathrm{AKI}^{18,34}$.

Both APACHE II and GCS scoring systems are commonly used to evaluate the severity of critically ill patients ${ }^{35}$. However, in our study, APACHE II score but not GCS score, is independently associated with postoperative AKI. As APACHE II score is a physiologically based system containing 12 physiological parameters, it is a useful prediction tool of hospital consequences, such as mortality and AKI, in critically ill patients ${ }^{36}$. Moreover, APACHE II score system includes GCS score, and thus, pathophysiological changes predicting in an organism after systemic insult could be illustrated comprehensively and systematically by the APACHE II scoring system. Therefore, this scoring system is thought to be superior to GCS for prediction of adverse outcome ${ }^{37}$. In the present study, APACHE II score, instead of GCS score, is an independent predictor of postoperative AKI, probably owing to the same reason.

The commonly prescribed medications that predispose to renal impairment were also analyzed in our study. Although it is well known that nephrotoxic drugs (nonsteroidal anti-inflammatory drug, angiotensin-converting enzyme inhibitor, angiotensin receptor blocker, immunosuppressant, aminoglycoside, vancomycin, acyclovir, or amphotericin) have kidney impairment effect, the use of these drugs was not associated with AKI in the present study. The reason that we could not get statistically significant conclusions may be attributed to a small numbers of patients using these drugs in our cohort. 
It is increasingly evident that even relatively modest kidney injury is independently associated with an increased risk for morbidity and mortality ${ }^{38,39}$. In comparison with non-AKI patients, we found that AKI patients was significantly associated with in-hospital and ICU mortality, and other adverse outcomes, which was consistent with previous studies ${ }^{2,4,5,9,11}$. Although lacking effective therapy regimens at present, evaluating modifiable risk factors for AKI may contribute to developing novel strategies for preventing postoperative AKI in neurosurgical critically ill patients. Hence we attempted to find out modifiable predict factors predisposing to AKI. Of note, all aforementioned identified risk factors in our study are potential modifiable. Further intervention study should be performed to prove effectiveness regarding these modifiable risk factors before translating our research into clinical application.

There are several limitations that should be addressed in the present study. Similar to all observational research, the variables chosen as possible risk factors were based on available literature and investigator's hypotheses, but the effects of residual or unmeasured confounders on the observed associations between the risk factors and AKI cannot be ruled out. Furthermore, the follow-up duration was limited to the period of hospitalization, and thus, post-discharge outcomes could not be accounted for in the present analysis. Additionally, due to a small number of events, statistical power was limited to detect the impact of AKI on mortality, which is a hard endpoint. This may limit the ability to interpret our data on mortality. Last but not least, almost $74 \%$ of the patients in our cohort have had surgery for intracranial tumor. Therefore, the generalization of our conclusion to all neurosurgical critically ill patients may be limited. However, this disproportionate size of the intracranial tumor group reflected the real situation of three ICUs in the present study.

In conclusion, the present study showed that postoperative AKI based on KDIGO criteria occurred in $13.5 \%$ of neurosurgical critically ill patients. Independent risk factors for postoperative AKI were intraoperative estimated blood loss, postoperative reoperation, postoperative concentration of serum CysC, use of mannitol during operation, and postoperative APACHE II score. Postoperative AKI was closely related to adverse in-hospital outcomes. Therapies aimed at mitigating these risk factors may offer protection against this complication. Thus, this study could have significant clinical implications for neurosurgical critically ill patients at risk for postoperative AKI.

\section{Methods}

Study Design and Participants. The prospective observational study was conducted in the neurosurgical ICUs of three tertiary care hospitals in China. Patients aged 18 years or older who underwent neurosurgical procedure and admitted to the neurosurgical ICUs between January 2015 and April 2016 were included. The exclusion criteria included refusal of consent, preexisting end-stage renal disease or renal dysfunction requiring RRT before operation, preexisting renal transplantation, preexisting nephrectomy, presence of AKI before neurosurgical procedure, or missing admission data. The primary outcome was the occurrence of AKI within 7 days after neurosurgical operation. The protocol was in accordance with STROBE (Strengthening the Reporting of Observational Studies in Epidemiology) guidelines ${ }^{40}$. All experiments were performed in accordance with the approved protocols, guidelines, and regulations. This study was approved by the Ethics Committee of the Guangdong General Hospital, as well as the committees of other two participating centers (the Ethics Committee of Xiaolan Hospital of Southern Medical University and the Ethics Committee of Guangzhou Nansha Central Hospital), and all patients (or appropriate surrogates for patients unable to consent) provided written informed consent.

Data Collection. Each patient's clinical data were prospectively collected from electronic hospital and laboratory databases. The following variables were recorded: age, gender, BMI, preexisting clinical conditions [hypertension, diabetes mellitus, CKD, cerebrovascular disease, hyperlipidemia, and coronary artery disease (CAD)], prior neurological surgery, emergency surgery, ASA classification, preoperative use of nephrotoxic drugs (nonsteroidal anti-inflammatory drug, angiotensin-converting enzyme inhibitor, angiotensin receptor blocker, immunosuppressant, aminoglycoside, vancomycin, acyclovir, or amphotericin), preoperative administration of radiographic contrast or mannitol, and diagnostic group (spine disease, hydrocephalus, intracranial tumor, traumatic brain injury, intracranial aneurysm/arteriovenous malformation, hypertensive cerebral hemorrhage, or others). Laboratory values were obtained, including level of preoperative hemoglobin, baseline serum creatinine, and concentration of postoperative serum creatinine, hemoglobin, and CysC at ICU admission. Blood samples were measured at the central laboratory of the Guangdong General Hospital using a standard protocol. Serum creatinine and hemoglobin were measured before operation, and then measured after operation at ICU admission, and thereafter at least once a day as a part of routine clinical care during ICU hospitalization. Postoperative serum CysC was measured only once at ICU admission. We also recorded the hourly urine output of each patient from enrollment to ICU discharge. The baseline eGFR was calculated by the CKD-Epidemiology Collaboration Equation $^{41}$. The APACHE II score ${ }^{42}$ and the GCS $^{43}$ score were used to evaluate disease severity. The postoperative APACHE II and GCS scores were assessed immediately after patients had recovered from anesthetic. Postoperative reoperation within 7 days after first neurosurgical procedure was recorded.

Surgical data including duration of surgery, intraoperative estimated blood loss, lowest mean arterial pressure (MAP; i.e. lowest MAP for at least 5 continuous minutes) during anesthesia, amount and type of intraoperative fluids administered (crystalloid and colloid), intraoperative use of mannitol and transfusions (red blood cells, platelets, and plasma) were recorded. Artificial colloid solutions used during the study period consisted of the hydroxyethyl starch and gelatin.

Outcome variables were also recorded, including duration of postoperative mechanical ventilation, incidence of postoperative tracheal reintubation, tracheotomy and RRT, total ICU costs, ICU and in-hospital mortality, and length of stay in hospital and ICU. 
Definitions. AKI was defined based on the KDIGO criteria ${ }^{44}$ for AKI within one week after surgery as any of the following: increase in $\mathrm{sCr}$ by $\geq 0.3 \mathrm{mg} / \mathrm{dl}(\geq 26.5 \mu \mathrm{mol} / \mathrm{l})$ within 48 hours, or increase in $\mathrm{sCr}$ to $\geq 1.5$ times baseline within one week, or urine output $<0.5 \mathrm{ml} / \mathrm{kg} / \mathrm{h}$ for 6 hours. AKI is staged according to the following KDIGO criteria. Stage 1: increase of sCr to 1.5-1.9 times from baseline, or $\geq 0.3 \mathrm{mg} / \mathrm{dl}(\geq 26.5$ $\mu \mathrm{mol} / \mathrm{l}$ ) increase of sCr, or urine output $<0.5 \mathrm{ml} / \mathrm{kg} / \mathrm{h}$ for $6-12 \mathrm{~h}$. Stage 2 : increase of sCr to $2.0-2.9$ times from baseline or urine output $<0.5 \mathrm{ml} / \mathrm{kg} / \mathrm{h}$ for $\geq 12 \mathrm{~h}$. Stage 3: 3 times increase of sCr from baseline or $\geq 4.0 \mathrm{mg} / \mathrm{dl}(\geq 353.6 \mu \mathrm{mol} / \mathrm{l})$ increase of $\mathrm{sCr}$ or initiation of RRT, or urine output $<0.3 \mathrm{ml} / \mathrm{kg} / \mathrm{h}$ for $\geq 24 \mathrm{~h}$ or anuria for $\geq 12 \mathrm{~h}$.

A baseline creatinine was determined by using the following rules ranked in descending order of preference as previously described: ${ }^{45}$ (1) the most recent pre-ICU value between 30 and 365 days before ICU admission $(\mathrm{n}=54)$; (2) a stable pre-ICU value $>365$ days for patients aged $<40$ years, (stable defined as within $15 \%$ of the lowest ICU measurement) before ICU admission $(n=1)$; (3) pre-ICU value $>365$ days before ICU admission and less than the initial serum creatinine on ICU admission $(\mathrm{n}=4)$; (4) a pre-ICU value (between 3 and 39 days before ICU admission) less than or equal to the initial on-admission serum creatinine to ICU and not obviously in AKI ( $n=352)$; (5) the lowest of initial on-admission serum creatinine to ICU $(n=150)$, the last ICU value $(n=50)$, or the minimum value at follow-up to 365 days $(n=13)$.

Statistical Methods. As reported previously described by Harrell, Vittinghoff, Steyerberg ${ }^{46}$, events per variable (EPV) $>10$ was an important issue for estimation of multivariable regression coefficients. To avoid biased estimation of regression Coefficients, EPV $=15$ would be required of interest in our final outcome model. Thus, to fit a model with 5 covariates, we would require approximately 75 outcome events. We calculated the sample size based on an estimated AKI incidence of $13 \%$, which we determined in a chart review of 100 patients undergoing neurosurgical operation (unpublished). Therefore, a sample size of 578 cases was required. Considering a possible dropout rate of $10 \%$, we would need approximately 636 cases.

Continuous variables were expressed as medians with interquartile range or mean $\pm \mathrm{SD}$. Categorical variables were expressed as number (percentage). The non-normally distributed continuous variables were compared by Wilcoxon rank-sum test. To compare the categorical variables, Chi-square or Fisher's exact test was used.

Univariate and multivariate logistic regression was used to evaluate the relationship between postoperative $\mathrm{AKI}$ and perioperative risk factors. The clinical perioperative variables with $P<0.10$ in univariate analysis were included in multivariate analysis. Logistic multivariate forward stepwise (likelihood ratio) regression was then used to determine the most efficient predictors of AKI. Results are presented as ORs with 95\% confidence intervals $(\mathrm{CI})$. All the tests were two-tailed, and $P<0.05$ was considered as statistical significance. SPSS version 13.0 were used.

\section{References}

1. Xu, X. et al. Epidemiology and Clinical Correlates of AKI in Chinese Hospitalized Adults. Clin J Am Soc Nephrol. 10(9), 1510-1518 (2015).

2. Bell, S. et al. Risk of postoperative acute kidney injury in patients undergoing orthopaedic surgery-development and validation of a risk score and effect of acute kidney injury on survival: observational cohort study. Bmj. 351, h5639 (2015).

3. Ohno, Y. et al. Impact of periprocedural bleeding on incidence of contrast-induced acute kidney injury in patients treated with percutaneous coronary intervention. J Am Coll Cardiol. 62(14), 1260-1266 (2013).

4. Karkouti, K. et al. Acute kidney injury after cardiac surgery: focus on modifiable risk factors. Circulation. 119(4), 495-502 (2009).

5. Abelha, F. J., Botelho, M., Fernandes, V. \& Barros, H. Determinants of postoperative acute kidney injury. Crit Care. 13(3), R79 (2009).

6. Huber, M. et al. Cardiovascular-Specific Mortality and Kidney Disease in Patients Undergoing Vascular Surgery. JAMA Surg. 151(5), $441-450$ (2016).

7. Grams, M. E. et al. Acute Kidney Injury After Major Surgery: A Retrospective Analysis of Veterans Health Administration Data. Am J Kidney Dis. 67(6), 872-880 (2016).

8. Romagnoli, S. \& Ricci, Z. Postoperative acute kidney injury. Minerva Anestesiol. 81(6), 684-696 (2015).

9. Perez-Valdivieso, J. R., Monedero, P., Vives, M., Garcia-Fernandez, N. \& Bes-Rastrollo, M. Cardiac-surgery associated acute kidney injury requiring renal replacement therapy. A Spanish retrospective case-cohort study. BMC Nephrol. 10, 27 (2009).

10. Haase, M. et al. A comparison of the RIFLE and Acute Kidney Injury Network classifications for cardiac surgery-associated acute kidney injury: a prospective cohort study. J Thorac Cardiovasc Surg. 138(6), 1370-1376 (2009).

11. Kheterpal, S. et al. Predictors of postoperative acute renal failure after noncardiac surgery in patients with previously normal renal function. Anesthesiology. 107(6), 892-902 (2007)

12. Thakar, C. V., Kharat, V., Blanck, S. \& Leonard, A. C. Acute kidney injury after gastric bypass surgery. Clin J Am Soc Nephrol. 2(3), 426-430 (2007)

13. Bell, S. et al. Risk of AKI with gentamicin as surgical prophylaxis. J Am Soc Nephrol. 25(11), 2625-2632 (2014).

14. Zhao, J. Z., Zhou, L. F., Zhou, D. B., Tang, J. \& Zhang, D. The status quo of neurosurgery in China. Neurosurgery. 62(2), 516-20; discussion 20-21 (2008).

15. Steiger, H. J., Kramer, M. \& Reulen, H. J. Development of neurosurgery in Germany: comparison of data collected by polls for 1997, 2003, and 2008 among providers of neurosurgical care. World neurosurgery. 77(1), 18-27 (2012).

16. Kovacheva, V. P. et al. Acute Kidney Injury After Craniotomy Is Associated With Increased Mortality: A Cohort Study. Neurosurgery. 79(3), 389-396 (2016).

17. Rosner, M. H. \& Okusa, M. D. Acute kidney injury associated with cardiac surgery. Clin J Am Soc Nephrol. 1(1), 19-32 (2006).

18. Moore, E. M. et al. The incidence of acute kidney injury in patients with traumatic brain injury. Ren Fail. 32(9), 1060-1065 (2010).

19. Corral, L. et al. Impact of non-neurological complications in severe traumatic brain injury outcome. Crit Care. 16(2), R44 (2012).

20. Eriksson, M., Brattstrom, O., Martensson, J., Larsson, E. \& Oldner, A. Acute kidney injury following severe trauma: Risk factors and long-term outcome. J Trauma Acute Care Surg. 79(3), 407-412 (2015). 
21. Ahmed, M., Sriganesh, K., Vinay, B. \& Umamaheswara Rao, G. S. Acute kidney injury in survivors of surgery for severe traumatic brain injury: Incidence, risk factors, and outcome from a tertiary neuroscience center in India. Br J Neurosurg. 29(4), 544-548 (2015).

22. Ando, G. et al. Acute kidney injury after percutaneous coronary intervention: Rationale of the AKI-MATRIX (acute kidney injuryminimizing adverse hemorrhagic events by TRansradial access site and systemic implementation of angioX) sub-study. Catheter Cardiovasc Interv. 86(5), 950-957 (2015).

23. Nangaku, M. Chronic hypoxia and tubulointerstitial injury: a final common pathway to end-stage renal failure. J Am Soc Nephrol. 17(1), 17-25 (2006)

24. Koch, C. G. et al. Duration of red-cell storage and complications after cardiac surgery. N Engl J Med. 358(12), 1229-1239 (2008)

25. Moulton, M. J., Creswell, L. L., Mackey, M. E., Cox, J. L. \& Rosenbloom, M. Reexploration for bleeding is a risk factor for adverse outcomes after cardiac operations. J Thorac Cardiovasc Surg. 111(5), 1037-1046 (1996).

26. Tsigou, E., Psallida, V., Demponeras, C., Boutzouka, E. \& Baltopoulos, G. Role of new biomarkers: functional and structural damage. Crit Care Res Pract. 2013;2013:361078.

27. Charlton, J. R., Portilla, D. \& Okusa, M. D. A basic science view of acute kidney injury biomarkers. Nephrol Dial Transplant. 29(7), 1301-1311 (2014).

28. Nejat, M., Pickering, J. W., Walker, R. J. \& Endre, Z. H. Rapid detection of acute kidney injury by plasma cystatin C in the intensive care unit. Nephrol Dial Transplant. 25(10), 3283-3289 (2010).

29. Bell, M. et al. Cystatin C is correlated with mortality in patients with and without acute kidney injury. Nephrol Dial Transplant. 24(10), 3096-3102 (2009)

30. Herget-Rosenthal, S. et al. Early detection of acute renal failure by serum cystatin C. Kidney Int. 66(3), 1115-1122 (2004).

31. Fang, L. et al. Mannitol is an independent risk factor of acute kidney injury after cerebral trauma: a case-control study. Ren Fail. 32(6), 673-679 (2010).

32. Yang, B. et al. Intravascular administration of mannitol for acute kidney injury prevention: a systematic review and meta-analysis. PLoS One. 9(1), e85029 (2014).

33. Kim, M. Y. et al. Increased risk of acute kidney injury associated with higher infusion rate of mannitol in patients with intracranial hemorrhage. J Neurosurg. 120(6), 1340-1348 (2014).

34. Li, N., Zhao, W. G. \& Zhang, W. F. Acute kidney injury in patients with severe traumatic brain injury: implementation of the acute kidney injury network stage system. Neurocrit Care. 14(3), 377-381 (2011).

35. Chesnut, R. M. Glasgow Coma Score versus severity systems in head trauma. Crit Care Med. 26(1), 10-11 (1998).

36. Siew, E. D. et al. Urine neutrophil gelatinase-associated lipocalin moderately predicts acute kidney injury in critically ill adults. $J$ Am Soc Nephrol. 20(8), 1823-1832 (2009).

37. Caronna, J. J. \& Stubgen, J. P. Predicting mortality in intensive care unit patients with stroke. Crit Care Med. 28(5), 1656-1657 (2000).

38. Chertow, G. M., Burdick, E., Honour, M., Bonventre, J. V. \& Bates, D. W. Acute kidney injury, mortality, length of stay, and costs in hospitalized patients. J Am Soc Nephrol. 16(11), 3365-3370 (2005).

39. Lassnigg, A. et al. Minimal changes of serum creatinine predict prognosis in patients after cardiothoracic surgery: a prospective cohort study. J Am Soc Nephrol. 15(6), 1597-1605 (2004).

40. von Elm, E. et al. The Strengthening the Reporting of Observational Studies in Epidemiology (STROBE) statement: guidelines for reporting observational studies. Ann Intern Med. 147(8), 573-577 (2007).

41. Levey, A. S. et al. A new equation to estimate glomerular filtration rate. Ann Intern Med. 150(9), 604-612 (2009).

42. Knaus, W. A., Draper, E. A., Wagner, D. P. \& Zimmerman, J. E. APACHE II: a severity of disease classification system. Crit Care Med. 13(10), 818-829 (1985).

43. Jennett, B., Teasdale, G., Braakman, R., Minderhoud, J. \& Knill-Jones, R. Predicting outcome in individual patients after severe head injury. Lancet. 1(7968), 1031-1034 (1976).

44. Kidney Disease: Improving Global Outcomes (KDIGO). Acute Kidney Injury Work Group. KDIGO Clinical Practice Guideline for Acute Kidney Injury. Kidney Int Suppl 2(Suppl 1), 1-138 (2012).

45. Endre, Z. H. et al. Early intervention with erythropoietin does not affect the outcome of acute kidney injury (the EARLYARF trial). Kidney Int. 77(11), 1020-1030 (2010).

46. Steyerberg, E. W., Schemper, M. \& Harrell, F. E. Logistic regression modeling and the number of events per variable: selection bias dominates. J Clin Epidemiol. 64(12), 1464-1465; author reply 3-4 (2011).

\section{Acknowledgements}

The authors would like to thank all the doctors, nurses, technicians, and patients involved at the three participating centers for their dedication in the study. The Office of Medical Science of the Guangdong General Hospital (Guangdong Academy of Medical Sciences) supervised the research, including the study design, protocol, ethical issues, data collection, and case report forms. We also thank the ethics committees of Xiaolan Hospital of Southern Medical University, Guangzhou Nansha Central Hospital for supervision and protocol approval. "Author Chunbo Chen" is currently receiving a grant (\#2014B020212023) from "the Science and Technology Planning Project of Guangdong Province, China (Key Program)"; "Author Yunjun Deng" is currently receiving a grant (\#2016A020215129) from "the Science and Technology Planning Project of Guangdong Province, China"; "Author Ruibin Chi" is currently receiving a grant (\#2016B1083) from “the Science and Technology Program of Zhongshan, China".

\section{Author Contributions}

Y.J.D. and J.Y. equally contributed to the design of the research and interpretation of the data; C.B.C., Y.J.D., and Y.Y.D. contributed to the conception/design of the research and interpretation of the data, and critically revised the manuscript. Y.J.D. and Z.Q.N. performed the statistical analysis. Y.J.D., J.Y., R.B.C., H.Y., D.Z., S.W., C.M., Z.Q.N., L.W., Y.L.Z., L.G., D.Q.Z., L.H.H., Y.Y.D., and C.B.C. contributed to the acquisition and analysis of the data, drafted the manuscript, agree to be fully accountable for ensuring the integrity and accuracy of the work. All authors have reviewed and approved this version of the manuscript.

Additional Information

Competing Interests: The authors declare that they have no competing interests.

Publisher's note: Springer Nature remains neutral with regard to jurisdictional claims in published maps and institutional affiliations. 
(i) Open Access This article is licensed under a Creative Commons Attribution 4.0 International License, which permits use, sharing, adaptation, distribution and reproduction in any medium or format, as long as you give appropriate credit to the original author(s) and the source, provide a link to the Creative Commons license, and indicate if changes were made. The images or other third party material in this article are included in the article's Creative Commons license, unless indicated otherwise in a credit line to the material. If material is not included in the article's Creative Commons license and your intended use is not permitted by statutory regulation or exceeds the permitted use, you will need to obtain permission directly from the copyright holder. To view a copy of this license, visit http://creativecommons.org/licenses/by/4.0/.

(C) The Author(s) 2017 